\title{
Development of nuclear medicine in Africa
}

\author{
Anna Grigoryan $^{1} \cdot$ Salah Bouyoucef $^{2} \cdot$ Mike Sathekge $^{3}$ (D) Mariza Vorster $^{3}$ (C) $\cdot$ Pilar Orellana $^{4} \cdot$ Enrique Estrada $^{4}$. \\ Miriam Mikhail Lette ${ }^{4}(1) \cdot$ Olga Morozova $^{4}$. Olivier Pellet ${ }^{4} \cdot$ Diana Paez $^{4}(\mathbb{0}) \cdot$ Roberto C. Delgado Bolton $^{5}(\mathbb{0}$. \\ Francesco Giammarile ${ }^{1,4}$
}

Received: 2 August 2021 / Accepted: 8 October 2021 / Published online: 25 October 2021

(c) The Author(s) 2021

\section{Introduction}

Over the past 50 years, nuclear medicine and molecular imaging (NMMI) have grown tremendously across the world and now collectively play an important role in the elaboration and implementation of management strategies for prioritized public health issues [1, 2]. In many countries during this period, critical advances in technology, computing software, radiopharmacy and instrumentation have boosted the impact of NMMI in multiple medical fields. This heightened impact has led increasingly to awareness of nuclear medicine as fundamental in the diagnosis and treatment of many disorders and diseases [3-8].

As part of this momentum, during the last three decades the continent of Africa has shown consistent development of NMMI. However, upon close analysis of the progress of NMMI in Africa, we noticed that South Africa and North African countries have exhibited development profiles similar to those of the developed world [3-12]. In contrast, the growth of NMMI in many other countries has remained

Francesco Giammarile

f.giammarile@iaea.org

1 Nuclear Medicine and Diagnostic Imaging Section, Division of Human Health, Department of Nuclear Sciences and Applications, International Atomic Energy Agency, 1400 Vienna, Austria

2 Service de Médecine Nucléaire CHU Bab El Oued, Bab El Oued, Algeria

3 University of Pretoria and Steve Biko Academic Hospital, Pretoria, South Africa

4 TCAF Section, Division of Africa, Department of Technical Cooperation, International Atomic Energy Agency, 1400 Vienna, Austria

5 Department of Diagnostic Imaging (Radiology) and Nuclear Medicine, University Hospital San Pedro and Centre for Biomedical Research of La Rioja (CIBIR), Logroño, La Rioja, Spain rather limited to the expansion of basic clinical services provided by studies with ${ }^{99 \mathrm{~m}} \mathrm{Tc}$ and treatment with Iodine 131. The development of NMMI in the African continent has always been strongly linked to efficient existing technical cooperation with, and support in various forms provided by, the International Atomic Energy Agency (IAEA) [13]. At the end of 2019, data from 44 African countries which share technical cooperation programs with the IAEA showed that 29 countries have at least one unit of NMMI and 1 country has only an in vitro radio immunoassay (RIA) unit. The remaining 14 countries have started feasibility studies, or are going to do so, for the implementation of their first unit. Only 5 countries have their own postgraduate programs in nuclear medicine for physicians, and 22 countries started training nuclear physicians abroad after the year 2000 (17 of these countries after 2004). This might be one of the main reasons why, in Africa, 17 countries started their first nuclear medicine unit during the period 2004-2019 (Fig. 1).

\section{Past development of nuclear medicine in Africa}

As in the other regions of the world [14], nuclear medicine in Africa is associated with a long history in which multidisciplinary involvement from different fields such as physics, physiologists and clinicians contributed to the introduction of nuclear techniques in the medical world during the sixties. First, rectilinear scanners and planar gamma cameras were installed during the sixties and seventies in South Africa and in North African countries. This was followed, in those same leading countries during the seventies and early eighties, by use of the first radio-immunology assays and therapeutic applications with radioiodine for Grave's Disease and thyroid cancers. However, this introduction of nuclear medicine differed in various countries since radiology and physics were the "parents" of nuclear medicine in South Africa while hematology, endocrinology, biophysics and physiology were the first users of nuclear medicine techniques in North African countries. Successful widespread 


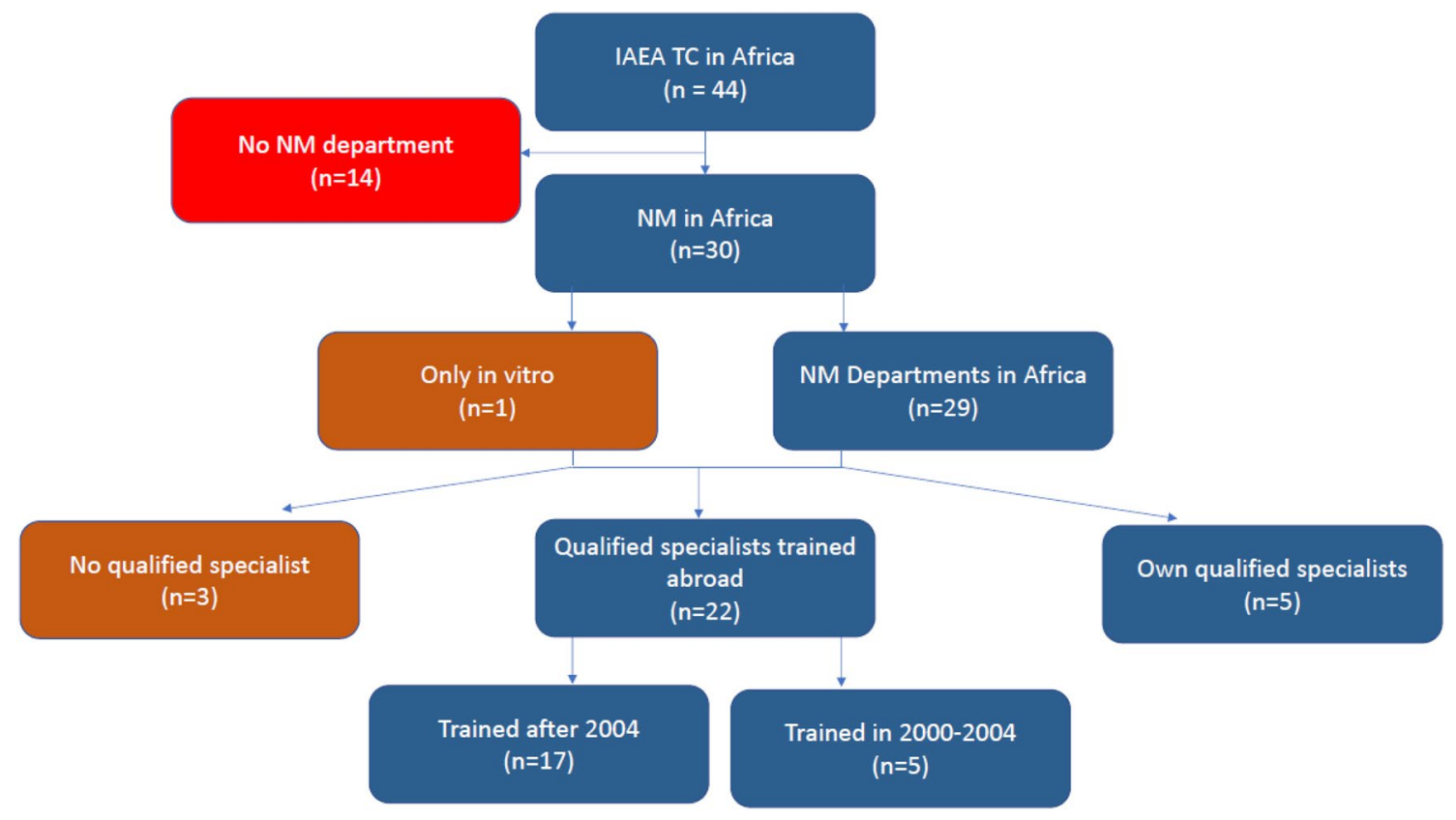

Fig. 1 Nuclear Medicine Situation in Africa

integration of nuclear medicine services and adoption by clinicians will continue to be strongly influenced by whether training was initiated at the undergraduate (preferable) or post-graduate level.

Later on, in the nineties, French-speaking countries of West Africa also followed the traditional development of nuclear medicine as had occurred in France by implanting their first nuclear medicine unit in the laboratories of biophysics located at a faculty of medicine [15]. Nuclear medicine became an independent specialty in some countries like Algeria (1985) and South Africa (1987). In others, nuclear medicine continues to keep formal links with other specialties like radiology (Egypt) and biophysics (Morocco and Tunisia). The first Single-Photon Emission Computed Tomography (SPECT) systems were introduced in the late seventies and early eighties in South Africa and in North Africa. This coincided with a boost in the practice of nuclear cardiology moving from planar to SPECT myocardial perfusion imaging [16]. Nuclear medicine started to mature in the early nineties when many departments opened as independent departments in several hospitals across Africa. Seeing the importance of the clinical impact and the potential applications of NMMI, health institutions in many countries in Africa promoted the further development and expansion of NMMI by implementing national commissions and regulations for the use of non-sealed sources in medicine and biology. In most instances, that step was achieved through the efficient cooperation and unique support of the IAEA. Many countries within the continent started to establish specific bilateral projects with the IAEA in nuclear medicine during the eighties and nineties which has boosted the widespread implementation of nuclear techniques in the medical field in Africa [3-8]. This period was of great importance since it directly induced in each country a clarification of the general clinical uses of ionizing radiation in medicine and therefore greatly contributed to distinguishing various relevant professionals in: nuclear medicine, radiation oncology, radiology, nuclear physics and biophysics. That step constituted an important turning point, since nuclear physicians started to be officially recognized as clinicians who deal with medical radioisotopes and are invited to participate in multidisciplinary medical boards $[17,18]$. Therefore, NMMI applications were clearly identified and integrated officially in the nomenclature of biological and medical acts.

\section{Nuclear medicine in Africa}

The global situation of Nuclear Medicine in Africa is illustrated in Table $1[19,20]$.

\section{Availability of radiopharmaceuticals}

The availability of diagnostic and therapeutic radiopharmaceuticals (RP) in Africa is very heterogenous. Infrequent and inconsistent supplies of RP are among the main factors which limit the development of nuclear medicine as well as the expansion of clinical nuclear medicine services in many countries [21-23]. There is only one country in the continent, South Africa, which has its own means of production 
Table 1 Nuclear Medicine professionals and equipment in Africa

\begin{tabular}{|c|c|c|c|c|c|c|c|c|c|c|}
\hline Countries & Population* & Income & NMP** & $\mathrm{MP}^{\dagger}$ & $\mathrm{NMT}^{\dagger \dagger}$ & $\mathrm{PET} / \mathrm{CT}$ & SPECT & Cyclotron & PET/M & SPECT/M \\
\hline Algeria & $43,851,044$ & Upper-Middle & 110 & 36 & 57 & 6 & 43 & 3 & 0.14 & 0.98 \\
\hline Angola & $32,866,272$ & Low & 3 & 0 & 1 & 0 & 1 & 0 & 0.00 & 0.04 \\
\hline Benin & $12,123,200$ & Low & 1 & 0 & 0 & 0 & 0 & 0 & 0.00 & 0.00 \\
\hline Botswana & $2,351,627$ & Upper-Middle & 1 & 0 & 0 & 0 & 1 & 0 & 0.00 & 0.45 \\
\hline Burkina Faso & $20,903,273$ & Low & 4 & 2 & 2 & 0 & 1 & 0 & 0.00 & 0.05 \\
\hline Burundi & $11,890,784$ & Low & 0 & 0 & 0 & 0 & 0 & 0 & 0.00 & 0.00 \\
\hline Cameroon & $26,545,863$ & Low & 2 & 2 & 0 & 0 & 1 & 0 & 0.00 & 0.04 \\
\hline RC Afrique & $4,829,767$ & Low & 0 & 0 & 0 & 0 & 0 & 0 & 0.00 & 0.00 \\
\hline Chad & $16,425,864$ & Low & 0 & 0 & 0 & 0 & 0 & 0 & 0.00 & 0.00 \\
\hline Congo DM & $86,790,567$ & Low & 3 & 0 & 4 & 0 & 1 & 0 & 0.00 & 0.01 \\
\hline Congo RE & $5,518,087$ & Low & 2 & 0 & 0 & 0 & 0 & 0 & 0.00 & 0.00 \\
\hline Côte d'Ivoire & $26,378,274$ & Low & 4 & 0 & 3 & 0 & 1 & 0 & 0.00 & 0.04 \\
\hline Djibouti & 988,346 & Low-Middle & 0 & 0 & 0 & 0 & 0 & 0 & 0.00 & 0.00 \\
\hline Egypt & $103,334,404$ & Upper-Middle & 105 & 105 & 95 & 18 & 74 & 6 & 0.18 & 0.74 \\
\hline Eritrea & $3,546,421$ & Low-Middle & 0 & 1 & 0 & 0 & 0 & 0 & 0.00 & 0.00 \\
\hline eSwatini & $1,160,164$ & Low-Middle & 0 & 0 & 0 & 0 & 0 & 0 & 0.00 & 0.00 \\
\hline Ethiopia & $114,963,588$ & Low-Middle & 1 & 4 & 3 & 0 & 3 & 0 & 0.00 & 0.03 \\
\hline Gabon & $2,225,734$ & Upper-Middle & 1 & 2 & 2 & 0 & 2 & 0 & 0.00 & 0.92 \\
\hline Ghana & $31,072,940$ & Low-Middle & 3 & 6 & 4 & 0 & 2 & 0 & 0.00 & 0.07 \\
\hline Kenya & $53,771,296$ & Low & 3 & 3 & 6 & 1 & 2 & 1 & 0.02 & 0.04 \\
\hline Lesotho & $2,142,249$ & Low-Middle & 0 & 0 & 0 & 0 & 0 & 0 & 0.00 & 0.00 \\
\hline Liberia & $5,057,681$ & Low & 0 & 0 & 0 & 0 & 0 & 0 & 0.00 & 0.00 \\
\hline Libya & $6,871,292$ & Upper-Middle & 10 & 0 & 10 & 1 & 5 & 1 & 0.15 & 0.74 \\
\hline Madagascar & $27,691,018$ & Low-Middle & 1 & 2 & 2 & 0 & 1 & 0 & 0.00 & 0.04 \\
\hline Malawi & $19,129,952$ & Low & 0 & 0 & 0 & 0 & 0 & 0 & 0.00 & 0.00 \\
\hline Mali & $20,250,833$ & Low & 1 & 1 & 2 & 0 & 1 & 0 & 0.00 & 0.05 \\
\hline Mauritania & $4,649,658$ & Low & 2 & 3 & 3 & 0 & 2 & 0 & 0.00 & 0.46 \\
\hline Mauritius & $1,271,768$ & High & 2 & 3 & 5 & 0 & 2 & 0 & 0.00 & 2.41 \\
\hline Morocco & $36,910,560$ & Upper-Middle & 65 & 28 & 40 & 12 & 28 & 2 & 0.33 & 0.77 \\
\hline Mozambique & $31,255,435$ & Low & 0 & 2 & 0 & 0 & 0 & 0 & 0.00 & 0.00 \\
\hline Namibia & $2,540,905$ & Upper-Middle & 4 & 6 & 4 & 0 & 6 & 0 & 0.00 & 2.41 \\
\hline Niger & $24,206,644$ & Low & 2 & 2 & 4 & 0 & 2 & 0 & 0.00 & 0.01 \\
\hline Nigeria & $206,139,589$ & Low & 3 & 9 & 9 & 0 & 3 & 0 & 0.00 & 0.02 \\
\hline Rwanda & $12,952,218$ & Low-Middle & 0 & 0 & 0 & 0 & 0 & 0 & 0.00 & 0.00 \\
\hline Senegal & $16,743,927$ & Low-Middle & 3 & 2 & 2 & 0 & 2 & 0 & 0.00 & 0.12 \\
\hline Seychelles & 98,347 & Upper-Middle & 0 & 0 & 0 & 0 & 0 & 0 & 0.00 & 0.00 \\
\hline Sierra Leone & $7,976,983$ & Low & 2 & 0 & 0 & 0 & 0 & 0 & 0.00 & 0.00 \\
\hline South Africa & $59,308,690$ & Upper-Middle & 65 & 185 & 100 & 21 & 86 & 3 & 0.24 & 1.47 \\
\hline Sudan & $43,849,260$ & Low-Middle & 3 & 11 & 19 & 0 & 7 & 0 & 0.00 & 0.16 \\
\hline Tanzania & $59,734,218$ & Low-Middle & 5 & 3 & 6 & 0 & 3 & 0 & 0.00 & 0.05 \\
\hline Togo & $8,278,724$ & Low-Middle & 1 & 0 & 0 & 0 & 0 & 0 & 0.00 & 0.00 \\
\hline Tunisia & $11,818,619$ & Upper-Middle & 60 & 17 & 50 & 4 & 16 & 1 & 0.26 & 1.37 \\
\hline Uganda & $45,741,007$ & Low & 2 & 0 & 3 & 0 & 1 & 0 & 0.00 & 0.02 \\
\hline Zambia & $18,383,955$ & Low & 3 & 4 & 3 & 0 & 2 & 0 & 0.00 & 0.11 \\
\hline Zimbabwe & $14,862,924$ & Low & 2 & 3 & 5 & 0 & 2 & 0 & 0.00 & 0.14 \\
\hline Total & $1086,693,675$ & & 479 & 442 & 444 & 63 & 301 & 17 & & \\
\hline
\end{tabular}

Italics: countries without in vivo NM equipment

*Population in 2019 [12]

**Nuclear medicine physicians

${ }^{\dagger}$ Medical physicist

${ }^{\dagger}$ Nuclear medicine technologist

${ }^{\ddagger}$ PET per million inhabitants

${ }^{*}$ SPECT per million inhabitants 
of radio isotopes and commercialization of radiopharmaceuticals. All other countries import their radiopharmaceuticals (mainly from Europe) and more recently from Turkey and South Africa. For decades, North African countries have had regular flights to Europe and Turkey and have relative advantages to import their radiopharmaceuticals. The limiting factor for buying radiopharmaceuticals from outside had often been the projected cost, misaligned with the allocatable budget. Clinical referrals to nuclear medicine services are the mainstay for establishing the budget, which may also be supported by revenues generated by nuclear medicine services. In fact, most countries in Africa are designated by the World Bank as low-income (LI) or low to middleincome (LMI). Within the NMMI context, this means that they have insufficient budgets for health services, including for buying radiopharmaceuticals regularly [2, 12, 24]. The variable availability of radiopharmaceuticals induces irregularities in nuclear medicine activities and therefore negatively impacts in clinical services [21]. As a consequence, departments have adopted a strategy of gathering patients during the period of availability of RP. Regarding radiopharmaceuticals used for SPECT imaging, all countries have ${ }^{99} \mathrm{Mo}-{ }^{99 m}$ Tc generators. $\mathrm{I}^{131}$ is widely available for both diagnostic and therapeutic purposes in countries which have infrastructure for therapeutic applications. Other radiopharmaceuticals which are relatively expensive or very specialized are most frequently used exclusively in North Africa and South Africa.

\section{Nuclear medicine facilities in Africa}

The situation of nuclear medicine equipment is available in IMAGINE website, the IAEA Medical imAGIng and Nuclear mEdicine global resources database, a snapshot of relevant infrastructure in over 170 countries and territories, launched in 2019 [17]. From the 30 countries with at least one nuclear medicine unit, one country has only an RIA unit with no access to nuclear medicine imaging. Among the 29 countries with nuclear medicine imaging, 16 have a unique department for the whole country while 13 have at least 2 nuclear medicine departments. It is clearly established and confirmed that the highest densities of nuclear medicine departments are in North Africa and South Africa. An impressive fact observed from the data is that, of the 29 countries with access to nuclear medicine, 18 started after 2004 and 4 started between 2000 and 2004. Three countries (Algeria, Egypt and South Africa) started SPECT nuclear medicine imaging during the eighties, 3 (Morocco, Tunisia and Namibia) during the nineties, and Libya in early 2000. 28 countries have implemented all their nuclear medicine facilities within hospitals or cancer centers while 2 countries have their facilities outside hospitals, the first at the faculty of medicine (Benin, RIA) and the second in an institute of radioisotopes which has a special health convention with a hospital (Niger).

\section{Conventional nuclear medicine equipment}

According to data provided to the IAEA, 297 SPECT Gamma cameras are available in 29 countries within Africa, with significant disparities between countries [1]. This translates into 0.24 SPECT scanners per million inhabitants, low as compared to other regions. Regarding the country income status, $85 \%$ of the total SPECTs (gamma cameras) available in the continent reside in 5 particular upper-middle income (UMIC) countries, South Africa and in North Africa. If we assess hybrid SPECT/CT scanners in Africa, almost 93\% have been installed in those 5 countries (Fig. 2).

\section{Cyclotrons and PET imaging}

In the region, there is great heterogeneity related with technical capacities available, with some countries having state-of-the-art technology including cyclotrons and Positron Emission Tomography hybridized with Computed Tomography (PET/CT), and other with no nuclear medicine units (Figs. 3 and 4). PET imaging is possible when Fluorodeoxyglucose (FDG) site of production is in the vicinity of the nuclear medicine service. In the world, the most common mode of production of Fluorine 18 is done through cyclotrons for which the power, the size and numbers are predetermined according to needs, market and the economy of the country [3-6, 25, 26]. Most of the time, private companies lead the production and delivery of FDG by investing in cyclotrons, radiopharmacy and quality control laboratories $[6,7,25]$. Due to the weak economy and the small market, private companies are reluctant to invest in LI and LMI countries [2]. Moreover, the cost of FDG is higher than the cost in HICs [2]. Seventeen cyclotrons supply 63 PET/CT cameras within the continent. Six countries concentrate all cyclotrons facilities which most of the time are managed by private companies. These facilities have contributed to the rapid local development of PET/CT in such countries particularly in Egypt [27], South Africa [28], and Morocco [16]. Other cyclotrons have been installed in Algeria, Tunisia and Kenya [19]. FDG constitutes by far the most common tracer for PET imaging [26, 29]. Less frequently, DOTATATE and PSMA labeled with Ga68 are used, most of the time in South Africa [28].

\section{Nuclear medicine therapy facilities}

Twenty-one countries practice nuclear medicine therapies. However, only 13 countries have facilities for inpatients while eight countries solely treat outpatients. Most of the facilities for therapy in Africa are concentrated in five 


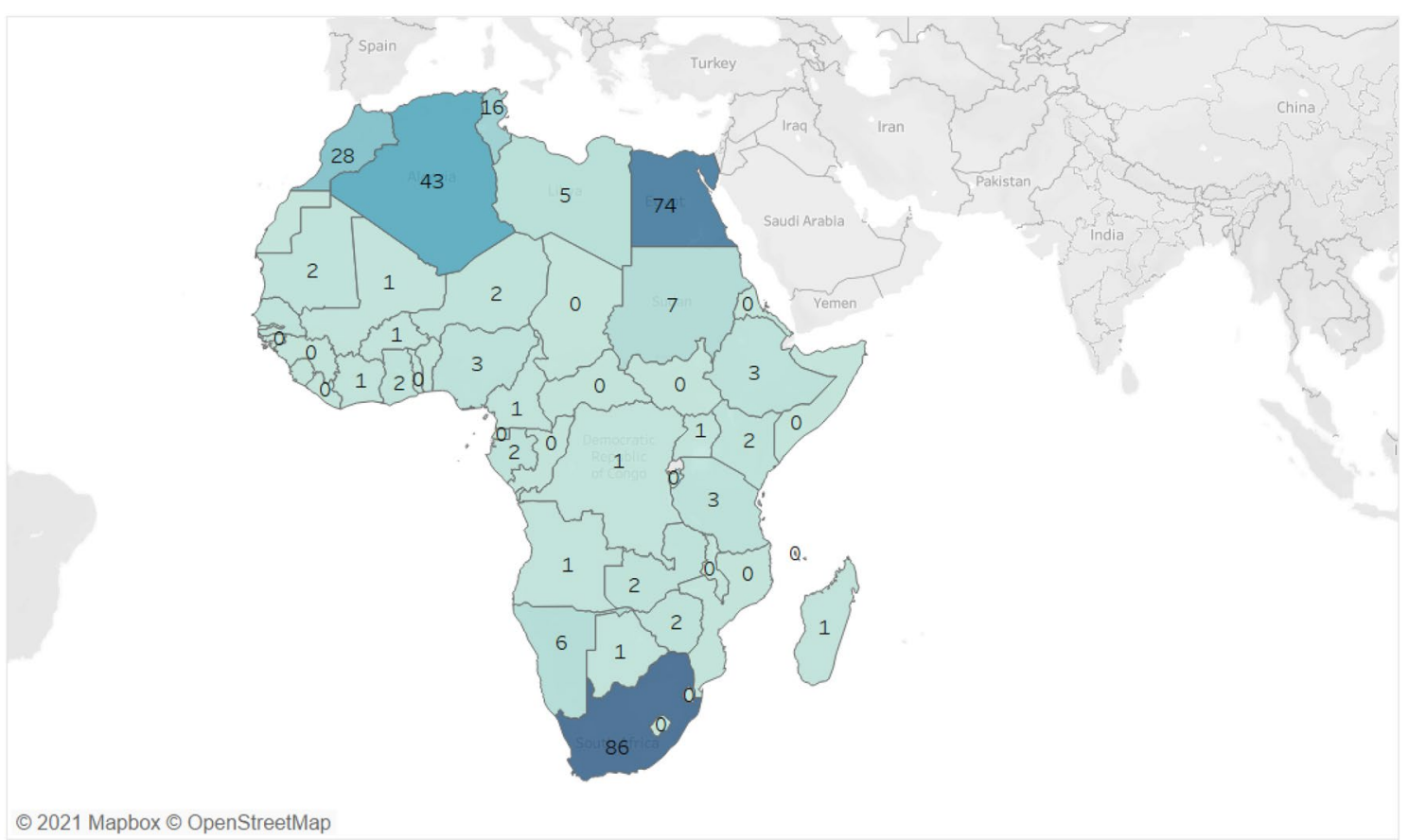

Total Number ..

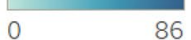

Fig. 2 Total numbers of conventional nuclear medicine equipment (i.e. SPECT)

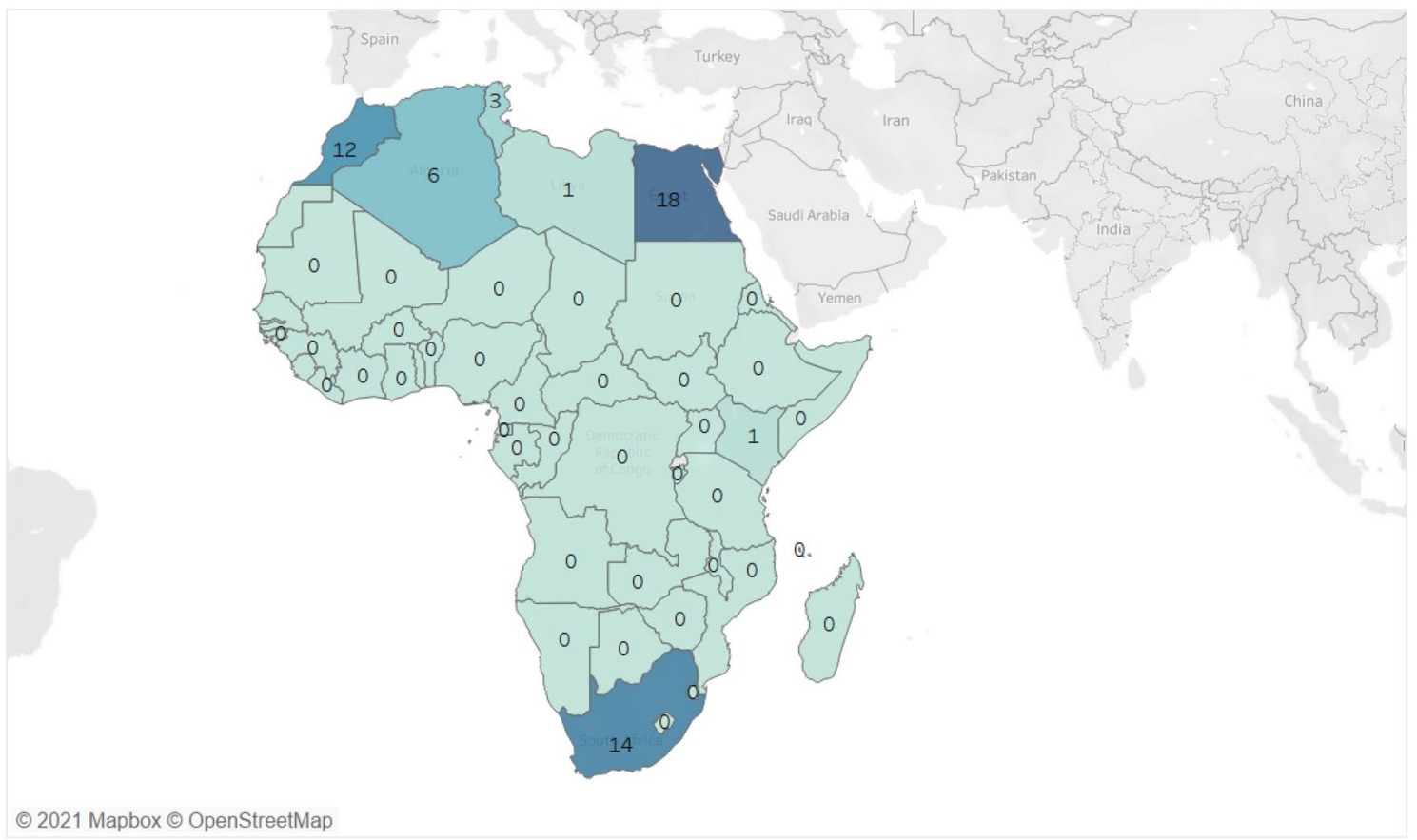

Total Number ..

18

Fig. 3 Total numbers of PET and PET/CT 


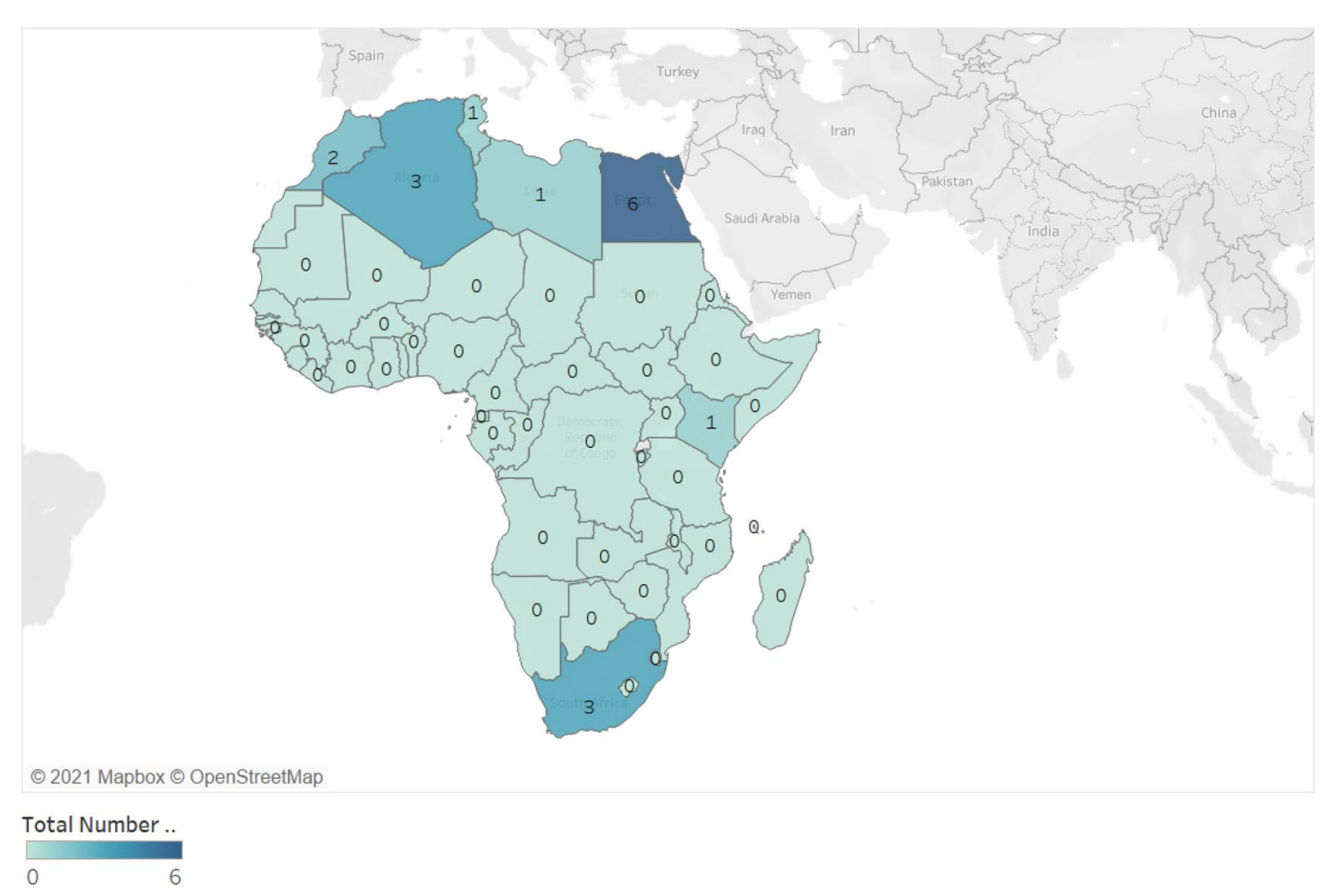

Fig. 4 Total number of cyclotrons

countries in North Africa, and South Africa. They have the densest and the oldest network of radionuclide therapy in Africa. The most common NM outpatient therapeutic procedure in Africa is ${ }^{131}$ I treatment of Grave's Disease, while the most common inpatient therapy is for thyroid cancer $[2,6]$.

Bone pain palliation with ${ }^{153} \mathrm{Sm}$ or ${ }^{89} \mathrm{Sr}$ is performed essentially in five countries in North Africa, and South Africa and ${ }^{131}$ I-MIBG is used in three countries in North Africa, and South Africa. ${ }^{90} \mathrm{Y}$ for radiosynoviorthesis is mainly available in some North African countries, and in South Africa. Therapies with ${ }^{177} \mathrm{Lu}$ are essentially limited to South Africa, while ${ }^{188} \mathrm{Re}$ and ${ }^{186} \mathrm{Re}$ have been clinically used in South Africa and Algeria. Finally, alpha emitters such as ${ }^{223} \mathrm{Ra}$ or ${ }^{225} \mathrm{Ac}$ are applied solely in South Africa [28] (Fig. 5).

\section{Human resources and education}

\section{Nuclear medicine physicians}

Thanks to cooperation with the IAEA for capacity building of human resources in NM, advancement in professional competencies observed during the past decades has been pronounced $[15,30]$. This has been pivotal to success in the development of nuclear medicine in Africa, which directly reflects the progressive availability of qualified staff working in nuclear medicine units (Fig. 6). Actually, 27 countries have at least one qualified nuclear medicine physician. 22 have seen their first qualified nuclear medicine physician since the year 2000, among them 17 countries after 2004 $[2,6,13]$. Five countries (Algeria, Egypt, Morocco, South Africa and Tunisia), where nuclear medicine is an independent specialty, have been offering both undergraduate and postgraduate training for nuclear physicians for the past 2-3 decades. Training consists of a 4-year program and is linked to obtaining a master's degree. The current availability of technical equipment and radiopharmaceuticals allows the development of more complete training. This explains the fact that $85 \%$ of the total number of nuclear physicians in Africa are working in those countries. The five countries have built their nuclear medicine infrastructure in both public and private sectors by recruiting their own locally educated skilled human resources, a big advantage compared to other countries which are experiencing shortages of qualified staff.

\section{Radiopharmacists or radiochemists}

Excluding the scarce number of radiopharmacists or radiochemists in the region, the specialty has grown in the last two decades. In the year 2000, South Africa was the sole country with a qualified radiopharmacist. Actually, eight 
Fig. 5 Nuclear medicine therapy activity in Africa per countries
Outpatient: activity only (thyroid)

Inpatient: basic (thyroid)

Inpatient: standard (e.g. bone pain palliation)

Inpatient: advanced (research)

No information available
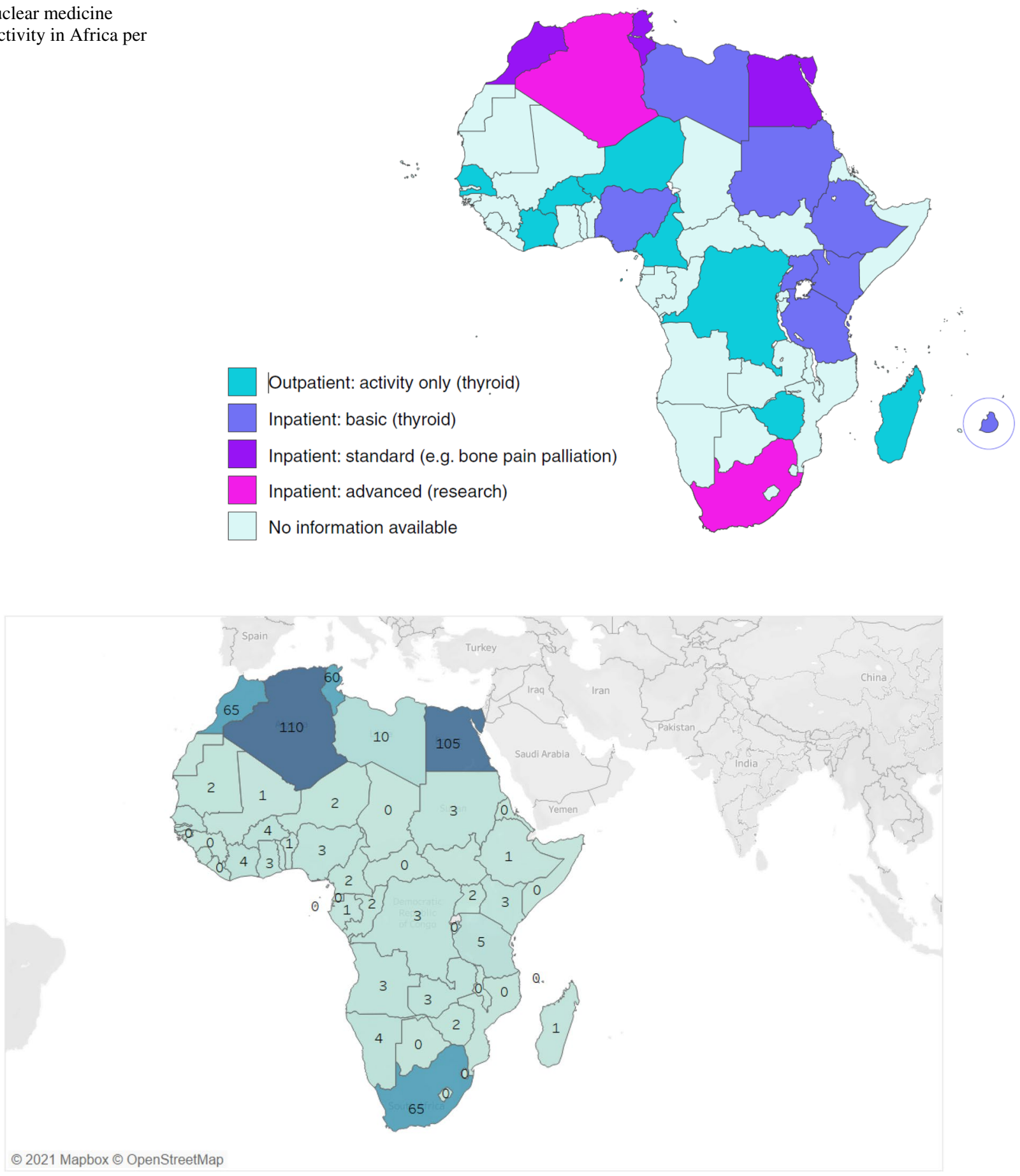

NM Physicians ..

$0 \quad 110$

Fig. 6 Total numbers of NM physicians per country

countries in Africa now have qualified radiopharmacists or radiochemists, who in most cases have trained abroad [ $2-5$, 15]. However, with growing research and development infrastructure in some countries, the need for training scholars with the highest academic degrees has arisen, and in certain countries, for example South Africa, training programs in radiopharmacy or radiochemistry already exist at $\mathrm{PhD}$ level. More recently, Morocco and Egypt introduced a master of 
radiopharmacy at the university level; while Algeria introduced radiopharmacy education as a subspecialty open for senior specialists. To fulfill the need for sustainable services at hospital radiopharmacy facilities, degrees at the technician level in radiopharmacy or radiochemistry, or onsite training for individuals who have obtained basic science degrees, are under preparation in some countries [22, 23].

\section{Medical physicists}

Medical physics related to nuclear medicine started in the eighties in Africa, when the first qualified medical physicists were recruited by hospitals in South Africa, Egypt and Algeria. Since the year 2000, technical cooperation with the IAEA has boosted the education and training of medical physicists in several countries, having achieved sustained success years later [2,13]. Fifteen countries have qualified medical physicists related to nuclear medicine and 8 countries (South Africa, Egypt, Algeria, Morocco, Tunisia, Sudan, Ghana, and Zimbabwe) have their own education system for MP.

The specific tasks of the physicists include QC testing of all cameras and radiopharmaceuticals. They manage most of the regulatory issues relating to radiation safety and function as the primary radiation protection officer for both patients and staff. They are also responsible for the training of radiographers and nursing staff with regards to radiation protection. In the majority of African countries, physicists are closely involved in the maintenance and repairs of equipment and in the motivation, procurement and in the setting up of specifications for new equipment. Additional responsibilities include ensuring that patients treated with radionuclide therapies are safely isolated and discharged and are consulted for dosimetry calculations, especially where such calculations affect further patient management.

With the emergence in some countries of master's degrees for nuclear medicine technologists, there is an overlap between tasks and responsibilities of the technologist and medical physicist which could lead in the figures to inappropriate numbers of MP. Traditionally, tasks of technologists are essential in the routine work of any department of nuclear medicine including the hot lab management, calibration of doses to be given to patients, use of gamma cameras, imaging of patients and applying safety rules. Meanwhile, the role of the medical physicist consists of different duties which are represented by radiation protection programs, quality control of NM instruments, optimization of NM clinical procedures, and monitoring radionuclide therapies.

\section{Technologists and nurses}

As radiographers for radiology, nuclear medicine technologists are a main pillar of any department of NMMI.
Education and training of technologists are essential for the quality of care provided by nuclear medicine services [31]. Six African countries are providing specialized training for technologists in nuclear medicine either at universities (Egypt, Sudan, and South Africa) or at dedicated schools for technologists and nurses (Algeria, Morocco, and Tunisia. The majority of qualified nuclear medicine technologists in Africa work in those 6 countries able to educate and train them. Many technologists working in nuclear medicine departments in sub-Saharan African countries do not have access to formal training in nuclear medicine and have been recruited mostly from related fields of radiologic technology. Because of the nature of the specialty, the numbers who require training are small, and it is therefore not costeffective for higher-education institutions in many countries to set up training programs. For that reason and in most of the cases, many countries in Africa have been encouraged by cooperation with the IAEA to train their technologists abroad within well-established countries, in Africa most of the time $[2,13]$. Several countries employ nurses in nuclear medicine departments, mostly those which have inpatient facilities. However, there is no specific education for nurses working in nuclear medicine and certain countries provide on-the-job training with emphasis on radiation safety and radionuclide therapies. Algeria has recently formed a working group to identify all tasks undertaken by nurses in nuclear medicine and compile a syllabus for a training program.

In case there are no technicians available, physicians could potentially inject and scan patients. This is often the situation in an after-hours setting and is less than ideal. Physicians are not experts in image acquisition and this practice would negatively impact on the number of patients that could be imaged.

\section{Critical role of the IAEA in the growth of nuclear medicine in Africa}

The history of development of nuclear medicine in Africa spotlights the unique role of the IAEA [2-5, 13, 30], an independent international organization in the United Nations family. The IAEA has a Department of Nuclear Sciences and Applications which helps countries to use nuclear and isotopic techniques to promote sustainable development objectives in agriculture, human health, water resource management, marine environment, and industrial applications $[1,13]$. The Human Health Program is overseen by the Division of Human Health and, within this division, the Nuclear Medicine and Diagnostic Imaging Section provides advice on establishing and operating nuclear medicine facilities and encourages appropriate use and quality management approaches for obtaining the best results from the practice of nuclear medicine [2]. IAEA cooperation with countries 
is based on technical cooperation projects which can be national, regional or interregional. Nuclear medicine constitutes one of the main focuses in human health cooperation programs developed by the IAEA and 29 countries in Africa presented specific projects related to nuclear medicine during the last two decades. Aims of those national projects range from the first introduction or expansion of nuclear medicine applications within a country or in a region of the country to the development of quality assurance programs or implementation of advanced nuclear medicine imaging like PET/CT [32-34]. Technical cooperation projects in Africa can be national, regional, interregional, or coordinated research projects.

National projects $[2,13]$ involve a single country and focus on supporting national development priorities where the use of nuclear medicine is an essential element for the achievement of national health objectives or provides a solution to a health problem in a cost-effective, safe and secure manner. The contents of the national projects include many aspects like providing education and training, equipment (gamma cameras, hot cells, dose calibrators...), supplies of radiopharmaceuticals and expertise. The impact of those national projects initiated by the division of Africa at the IAEA is huge with very tangible outcomes; for example, since 2004 more than 17 countries have started clinical nuclear medicine practices with the assistance of the IAEA, and 30 countries have extended their clinical nuclear medicine services thanks to those national projects. Hundreds of fellowships have been awarded over the last two decades by the IAEA to all categories of staff in Africa, some with long-term durations. The theoretical duration of a national project in nuclear medicine is 2 years. However, due to the dependence of the implementation rate on challenges faced, mostly locally, some national projects in nuclear medicine may last longer. The highest success rates of such national projects in nuclear medicine occur when the national health programs are temporally congruent.

Regional projects deliver technical cooperation support across national boundaries and address the needs in nuclear medicine of several Member States in Africa [2, 13]. Such projects consider national development objectives but are developed according to regional development priorities established by regional cooperative agreements, strategies and frameworks. Regional projects represent a unique way of networking for nuclear medicine professionals in the continent of Africa where the obvious lack of financial support or sponsorship reduces considerably the opportunities of meetings between nuclear physicians and other nuclear medicine staff. More than 12 regional projects directly or indirectly related to the development of clinical nuclear medicine in Africa were realized between 2000 and 2019. Those projects called RAF, with a mean duration of 3-5 years, play a major role in developing and sustaining nuclear medicine in Africa. Success of the regional projects has been evident. The coordinating meetings of these regional projects constitute a real "brainstorming" where all participants discuss major ideas, challenges, and victories related to the practice and to the development of nuclear medicine services in Africa. In addition, these coordinating meetings represent a stimulating opportunity for networking, expressing solidarity, and offering assistance to those countries which have less developed nuclear medicine services. On average two to three coordinating meetings are held during the lifetime of a regional project and permanent communication is established between all project coordinators as well as with the technical officer, the project manager, and the project scientific consultant. Among activities of the projects, the organization of week-long regional training courses (RTC) which focus upon one or two clinical and technological topics constitutes one of the main goals of the project. RTCs target nuclear medicine physicians, technologists, medical physicists and radiopharmacists and are organized each time in turn by different countries. RTCs gather on average 20-25 participants and are taught by high-level experts who provide up-to-date knowledge, through active learning and practical demonstrations. These courses are a unique, effective way to enhance local awareness of nuclear medicine. Experts and enthusiastic staff are convened from diverse African destinations in a forum where real exchanges of experiences take place and benefit both foreign and local participants, alike. More than 800 nuclear medicine physicians and 500 technologists have participated in these RTCs over the two last decades, taking in consideration that RTCs for radiopharmacists and medical physicists are covered under separate projects.

There are also a few inter-regional projects that deliver support across national and regional boundaries, and address the needs for nuclear medicine of several Member States in different regions [13]. They are categorized as transregional, global, capacity-building or as joint activities with an international entity. Africa is also participating in this type of project, the best example of which is the use of Quality Management Audits in Nuclear Medicine Practices (QUANUM), a tool which aims to improve quality of care delivered to patients [33-36].

Relevant global professional networking exists in Africa, mostly established through the IAEA meetings, and became with time an efficient and productive way of exchanging information and experiences between countries. Due to the insufficient performance of internet connections within Africa, few countries have successfully implemented telemedicine networks, as encouraged by the IAEA in Namibia and South Africa.

IAEA Coordinated Research Projects (CRPs) bring together scientists representing institutes in Member States to collaborate on a focused research topic that is of shared 
interest and is important to the mission of the Agency [37]. A CRP is normally approved for a period of 3-5 years and involves 8-15 laboratories, research teams or institutions. Most CRPs involve 2 or 3 Research Coordination Meetings (RCMs) where participants are brought together. The IAEA supports the cost of the RCMs, but only very limited funds are available for direct research support, and this only for participants from developing countries. Some departments of nuclear medicine within Africa are participating in CRPs where the research foci coincide directly with their local contexts.

\section{Recommendations}

Training can be coordinated in the continent and region with an institution like the Asian School of Nuclear Medicine, whereby the Colleges of Medicine of South Africa already have a structure that can be used for examination in English-speaking countries. Algiers could similarly facilitate examination in French-speaking countries. This can be done in conjunction with local universities and country regulators (medical councils). Such strategies can gain traction easily via a pre-existing link with The Association of Medical Councils of Africa (AMCOA). AMCOA is an Association of Medical Regulatory Authorities in Africa, with the primary purpose of supporting medical regulatory authorities in Africa in the protection of the public interest by promoting high standards of medical education, registration and regulation, and facilitating the ongoing exchange of information among medical regulatory authorities [38].

The association further exists to provide guidance to healthcare professional registered by Member States with a view to ensure the provision of quality healthcare by way of guidelines on various aspects of healthcare delivery such as setting standards for education and training, ethics guidelines, and encouraging continuous professional development among others [38].

Integration and working closely with the World Health Organization will help with prioritization of nuclear medicine with inclusion of our procedures in the essential drug list and thus necessitate routine budgets.

\section{Conclusion}

Progressive provision of nuclear medicine services in over 20 African countries has been the direct result of decades of fruitful cooperation between the IAEA and multiple African Member States. The expectation is that this trend continues with increasing contribution of African countries towards capacity building, maintenance and sustainability.
Hopefully the African population have more access to this lifesaving and rapidly expanding technology. Therefore, more SPECT and PET scanners shall be installed across Africa in coming decades, and more skilled professionals trained, matching increased integration of nuclear medicine in national healthcare system development. Partnerships with specialized organizations can provide important assistance in this regard as well as the collaboration of local government, business and medical entities in each country.

It is believed that the future for African Member States' Nuclear Medicine services is linked to an overall development of all vital components at the regional level through mainly focusing on a strategy that optimizes the transport of radiopharmaceuticals, tracers for therapy or for non-routine diagnosis even in countries that do not produce them.

In many African countries, yet to be realized is the longterm sustainable impact of incrementally implementing nuclear imaging and therapies, towards rationally addressing major population-based health problems faced by Africa [39].

\section{Declarations}

Conflict of interest The following authors declare no conflict of interest. Anna Grigoryan, Salah Bouyoucef, Mike Sathekge, Mariza Vorster, Pilar Orellana, Enrique Estrada, Miriam Mikhail Lette, Olga Morozova, Olivier Pellet, Diana Paez, Roberto C. Delgado Bolton, Francesco Giammarile.

Human and animal studies This article does not contain any studies with human or animal subjects performed by the any of the authors.

Open Access This article is licensed under a Creative Commons Attribution 4.0 International License, which permits use, sharing, adaptation, distribution and reproduction in any medium or format, as long as you give appropriate credit to the original author(s) and the source, provide a link to the Creative Commons licence, and indicate if changes were made. The images or other third party material in this article are included in the article's Creative Commons licence, unless indicated otherwise in a credit line to the material. If material is not included in the article's Creative Commons licence and your intended use is not permitted by statutory regulation or exceeds the permitted use, you will need to obtain permission directly from the copyright holder. To view a copy of this licence, visit http://creativecommons.org/licenses/by/4.0/.

\section{References}

1. Grossi RM (2021) The importance of medical imaging and nuclear medicine in universal health coverage. Lancet Oncol 22(4):423-424

2. Dondi M, Kashyap R, Paez D, Pascual T, Zaknun J, Bastos FM et al (2011) Trends in nuclear medicine in developing countries. J Nucl Med 52(Suppl 2):16S-23S

3. Lass P (2005) The situation of nuclear medicine in Central and Eastern Europe. Eur J Nucl Med Mol Imaging 32(12):B11-B14 
4. Paez D, Becic T, Bhonsle U, Jalilian AR, Nunez-Miller R, Osso JA Jr (2016) Current status of nuclear medicine practice in the Middle East. Semin Nucl Med 46(4):265-272

5. Paez D, Peix A, Orellana P, Vitola J, Mut F, Gutierrez C et al (2017) Current status of nuclear cardiology practice in Latin America and the Caribbean. J Nucl Cardiol 24(1):308-316

6. Lee DS, Lee YS, Lee JS, Suh MS (2019) Promotion of nuclear medicine-related sciences in developing countries. Nucl Med Mol Imaging 53(2):73-82

7. Delbeke D, Segall GM (2011) Status of and trends in nuclear medicine in the United States. J Nucl Med 52(Suppl 2):24S-S28

8. Paez D, Orellana P, Gutierrez C, Ramirez R, Mut F, Torres L (2015) Current status of nuclear medicine practice in Latin America and the Caribbean. J Nucl Med 56(10):1629-1634

9. Global Nuclear Medicine Market Research Report. Eurostat. Healthcare resource statistics. Technical Resources and Medical Technology Statistics Explained. 2020 [Available from: https:// ec.europa.eu/eurostat/statisticsexplained. Accessed 9 Sept 2021

10. IMV Medical Information. PET Imaging Market Summary Report. 2020 [Available from: https://imvinfo.com/product/2020pet-imaging-market-summaryreport/. Accessed 9 Sept 2021

11. Paez D, Giammarile F, Orellana P (2020) Nuclear medicine: a global perspective. Clin Transl Imaging 8(2):51-53

12. World Bank Group. World Bank Data 2021 [Available from: https://data.worldbank.org/indicator/SH.DTH.NCOM.ZS?view= chart. Accessed 9 Sept 2021

13. Casas-Zamora JA, Kashyap R (2013) The IAEA technical cooperation programme and nuclear medicine in the developing world: objectives, trends, and contributions. Semin Nucl Med 43(3):172-180

14. Mallard J, Trott NG (1979) Some aspects of the history of nuclear medicine in the United Kingdom. Semin Nucl Med 9(3):203-217

15. Gremillet E, Lemaire B, Prigent A, Vuillez JP (2013) Nuclear medicine training and practice in France. Eur J Nucl Med Mol Imaging 40(6):976-978

16. Bouyoucef SE, Mercuri M, Pascual TN, Allam AH, Vangu M, Vitola JV et al (2017) Nuclear cardiology practices and radiation exposure in Africa: results from the IAEA nuclear cardiology protocols study (INCAPS). Cardiovasc J Afr 28(4):229-234

17. Hricak H, Abdel-Wahab M, Atun R, Lette MM, Paez D, Brink JA et al (2021) Medical imaging and nuclear medicine: a lancet oncology commission. Lancet Oncol 22(4):e136-e172

18. Freudenberg LS, Paez D, Giammarile F, Cerci J, Modiselle M, Pascual TNB et al (2020) Global impact of COVID-19 on nuclear medicine departments: an international survey in April 2020. J Nucl Med 61(9):1278-1283

19. IAEA. IMAGINE - IAEA medical imaging and nuclear medicine global resources database 2021 [Available from: https://humanhealth. iaea.org/HHW/DBStatistics/IMAGINE.html. Accessed 9 Sept 2021

20. IAEA. Nuclear Medicine Database. NUMDAB. Human Health Campus Website. 2021 [Available from: https://nucmedicine.iaea. org/data. Accessed 9 Sept 2021

21. Kayode AP, Mertz C, Guyot JP, Brat P, Mouquet-Rivier C (2013) Fate of phytochemicals during malting and fermentation of type III tannin sorghum and impact on product biofunctionality. J Agric Food Chem 61(8): 1935-1942

22. IAEA. Operational Guidance on Hospital Radiopharmacy: A Safe and Effective Approach 2008 [Available from: https://www-pub. iaea.org/MTCD/Publications/PDF/Pub1342/Pub1342_web.pdf. Accessed 9 Sept 2021

23. Cutler CS, Bailey E, Kumar V, Schwarz SW, Bom HS, Hatazawa $\mathrm{J}$ et al (2021) Global issues of radiopharmaceutical access and availability: a nuclear medicine global initiative project. J Nucl Med 62(3):422-430

24. United Nations. Transforming Our World: The 2030 Agenda for Sustainable Development. 2016 [Available from: https://sdgs.un. org/2030agenda. Accessed 9 Sept 2021
25. Ell PJ (1993) The future of nuclear medicine: a perspective from Europe. J Nucl Med 34(1):169-170

26. Mettler FA Jr, Mahesh M, Bhargavan-Chatfield M, Chambers CE, Elee JG, Frush DP et al (2020) Patient exposure from radiologic and nuclear medicine procedures in the United States: procedure volume and effective dose for the period 2006-2016. Radiology 295(2):418-427

27. Einstein AJ, Pascual TN, Mercuri M, Karthikeyan G, Vitola JV, Mahmarian JJ et al (2015) Current worldwide nuclear cardiology practices and radiation exposure: results from the 65 country IAEA nuclear cardiology protocols cross-sectional study (INCAPS). Eur Heart J 36(26):1689-1696

28. Vorster M, Warwick J, Lawal IO, Du Toit P, Vangu M, Nyakale NE et al (2019) South African guidelines for receptor radioligand therapy (RLT) with Lu-177-PSMA in prostate cancer. S Afr J Surg 57(4):45-51

29. Einstein AJ (2020) Medical radiation exposure to the U.S. population: the turning tide. Radiology 295(2):428-429

30. IAEA (2019) TECDOC-1883, training curriculum for nuclear medicine physicians. Available from: https://www-pub.iaea.org/ MTCD/Publications/PDF/TE-1883web.pdf. Accessed 9 Sept 2021

31. McFarland GA, Johnson SG (2020) Nuclear medicine clinical practice in the united states during the COVID-19 era and beyond. J Nucl Med Technol 48(3):218-226

32. Gnanasegaran G, Paez D, Sathekge M, Giammarile F, Fanti S, Chiti A et al (2021) Coronavirus (COVID-19) pandemic mediated changing trends in nuclear medicine education and training: time to change and scintillate. Eur J Nucl Med Mol Imaging. https:// doi.org/10.1007/s00259-021-05241-2

33. Dondi M, Paez D, Torres L, Marengo M, Delaloye AB, Solanki $\mathrm{K}$ et al (2018) Implementation of quality systems in nuclear medicine: why it matters. An outcome analysis (quality management audits in nuclear medicine part III). Semin Nucl Med 48(3):299-306

34. Dondi M, Torres L, Marengo M, Massardo T, Mishani E, Van Zyl EA et al (2017) Comprehensive auditing in nuclear medicine through the international atomic energy agency quality management audits in nuclear medicine (QUANUM) program part 1: the quanum program and methodology. Semin Nucl Med 47(6):680-686

35. Dondi M, Torres L, Marengo M, Massardo T, Mishani E, Van Zyl EA et al (2017) Comprehensive auditing in nuclear medicine through the international atomic energy agency quality management audits in nuclear medicine program. Part 2: analysis of results. Semin Nucl Med 47(6):687-93

36. IAEA (2015) Quality Management Audits in Nuclear Medicine Practices. IAEA Human Health Series No. 33. 2nd edn. [Available from: https://www-pub.iaea.org/MTCD/Publications/PDF/Pub16 83Web-68161172.pdf. Accessed 9 Sept 2021

37. Duatti A (2008) Promoting nuclear medicine in developing countries through IAEA coordinated research projects: technical reports series 458 and 459. Q J Nucl Med Mol Imaging 52(3):209-211

38. Association of Medical Council of Africa. High Level Extract of Draft Strategic Plan 2018 - 20212021 [Available from: https://amcoa. org/amcoa-draft-strategic-plan-2018-2021/. Accessed 9 Sept 2021

39. (2021) Challenges and Promise for nuclear medicine in Africa: a conversation between Mike Sathekge and Johannes Czernin. J Nucl Med 62(5):593-595

Publisher's Note Springer Nature remains neutral with regard to jurisdictional claims in published maps and institutional affiliations. 